\title{
$\nabla$
}

\section{Sarcoidosis and chronic hepatitis C: treatment with prednisone and colchicine*}

\author{
Eduardo Guimarães Pereira ${ }^{1}$ \\ Caroline Bertolini Bottino ${ }^{1}$ \\ Ricardo Barbosa Lima ${ }^{1}$
}

\author{
Tais Ferreira Guimarães ${ }^{1}$ \\ Antonio Macedo D' Acri $^{1}$ \\ Carlos José Martins ${ }^{1}$
}

DOI: http:/ / dx.doi.org/10.1590/abd1806-4841.20164029

\begin{abstract}
Sarcoidosis is a disease which still has uncertain etiology. Possible environmental causes are cited in the literature, like organic and inorganic particles and infectious agents. Recent studies have demonstrated the occurrence of sarcoidosis in patients with chronic $\mathrm{C}$ hepatitis; however, this association remains without statistical or causal evidence. In this report a case of sarcoidosis associated with chronic hepatitis $C$ will be described, with subcutaneous lesions, considered rare, and good response to treatment with colchicine and prednisone. The hepatitis $C$ virus was isolated in sarcoid tissue and the association between the two diseases will be discussed.
\end{abstract}

Keywords: Giant cells; Hepatitis C, chronic; Sarcoidosis; Skin abnormalities

\section{INTRODUCTION}

Sarcoidosis is a multisystem disease, characterized by formation of noncaseating granulomas, with the skin being one of its preferred organs. ${ }^{1}$ First described in 1878 by Hutchinson, to this day it still has uncertain etiology and partially defined immunopathogenesis. ${ }^{1,2}$ Numerous environmental causes are cited in the literature as possible antigenic factors, with emphasis on organic and inorganic particles and infectious agents such as bacteria and viruses. ${ }^{1,3}$ It has a frequency of 10 to 40 cases for every 100,000 inhabitants, with important fluctuation according to the population studied. It portrays greater incidence in women, between the third and fourth decades of life and greater severity when it affects Afro-American individuals. ${ }^{1,3}$ It has a vast dermatologic presentation, taking the form of diverse elementary lesions, some of them of difficult diagnosis. Regarding the treatment, many drugs used did not go through controlled studies. ${ }^{3}$ Recent studies showed the concomitance of sarcoidosis in patients with Chronic Hepatitis C (CHC), like in the case reported.

\section{CASE REPORT}

Female patient, 50 years old, complained about dyspnea on moderate effort and dry cough for one year. At the dermatological examination she presented papules and erythematous infiltrated plaques on the face, back, shoulders and lower limbs, as well as nodules on elbows (Figure 1). She was empirically treated for pulmonary tuberculosis with RHZE scheme for four months and for hansenic reaction with Prednisone and thalidomide for 20 days, through primary health care, with no improvement. At that time, she was referred to the Dermatology service for diagnostic investigation. She reported being a $\mathrm{CHC}$ carrier for seven years, without specific antiviral treatment. Histopathological examination of the cutaneous lesion and subcutaneous nodule showed a sarcoid granulomatous infiltrate with giant cells and asteroid corpuscles (Figure 2). Staining for screening of infectious agents was negative. In CT of thorax were observed mediastinal lymphadenopathy and infiltrate in diffuse "frosted glass" pattern. Spirometry

Received on 13.09.2014.

Approved by the Advisory Board and accepted for publication on 16.10.2014.

Work performed at the Dermatology Service of Hospital Universitário Gaffrée e Guinle, Universidade Federal do Estado do Rio de Janeiro (HUGG - UNIRIO) - Rio de Janeiro (RJ), Brazil.

Financial Support: None.

Conflict of Interest: None.

1 Universidade Federal do Estado do Rio de Janeiro (UNIRIO) - Rio de Janeiro (RJ), Brazil.

(C)2016 by Anais Brasileiros de Dermatologia 

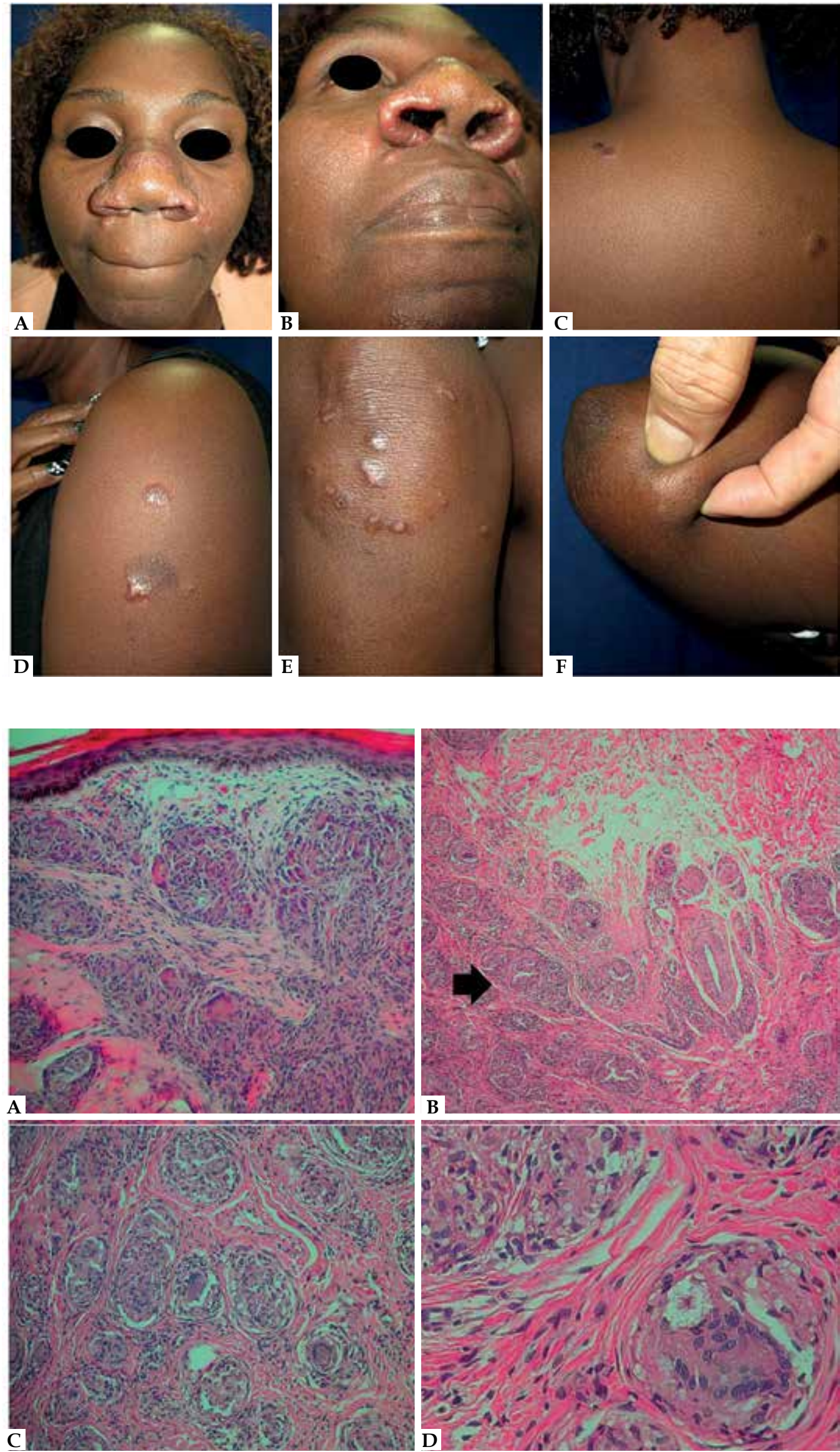

Figure 1: A and B) Papules and infiltrated plaques in the nasal region. C) Dorsal region. D) Left deltoid region. E) Right knee. F) Subcutaneous nodule on right elbow

Figure 2: A) Histology of skin showing sarcoid granulomatous infiltrate. (HE 100x) B) Histology of subcutaneous nodule showing infiltrate in hypodermis (arrow). (HE 40x) C) Sarcoid granulomas in the subcutaneous, with giant cells. (HE 100x) D) Asteroid corpuscle inside a giant cell. (HE 200x) 
showed mild restrictive ventilatory dysfunction. Angiotensin converting enzyme (ACE) serum levels were at the upper limit of normalcy. Tuberculin test (PPD) showed anergy. Considering these findings, the diagnosis of sarcoidosis was reached and treatment with prednisone $40 \mathrm{mg}$ /day and colchicine $1 \mathrm{mg} /$ day was established. After 30 days, improvement in facial lesions and lung infiltrate (thorax x-ray) was observed (Figure 3). Polymerase chain reaction (PCR) for HCV (hepatitis $C$ vírus) in sarcoid tissue was performed, with positive result.

\section{DISCUSSION}

As studies about immunopathogenesis of sarcoidosis advance, some questions arose regarding the associations previously considered fortuitous. Research has shown the involvement of specific antigenic factors which would lead to activation of T cells with production of cytokines characteristic of immune response Th1 in formation of granulomas, the basis of sarcoid involvement. This inappropriate response to an environmental factor would be the conjunction of exposure to a factor in a genetically predisposed individual, which was proven by the association of sarcoidosis with antigens encoded by HLA-DRB1 and HLA-DQ1. ${ }^{1,2,3}$

On the other hand, several diseases with different pathogenic mechanisms are associated with chronic infection by $\mathrm{HCV}$, among them cryoglobulinemia, glomerulonephritis, thyroiditis and lichen planus, suggesting that this infection is an important agent of different processes of immunological imbalance. ${ }^{4}$

In 1993, Blum and cols. published the first report on sarcoidosis in a patient with $\mathrm{CHC}$ and since then the number of published cases has been increasing. ${ }^{5}$ In 2005, in a review of 38 years of literature, RamosCasals and cols., identified 68 cases of sarcoidosis in individuals with $\mathrm{CHC}$. Of these, 50 cases were in individuals treated with interferon (INF), and only 18 in untreated individuals, such as in the case reported..$^{5}$ In this review, it was estimated that 90 cases of association of sarcoidosis and $\mathrm{CHC}$ have been described to the present day. ${ }^{5,6,7}$ It is suggested that $\mathrm{HCV}$, in genetically predisposed individuals,
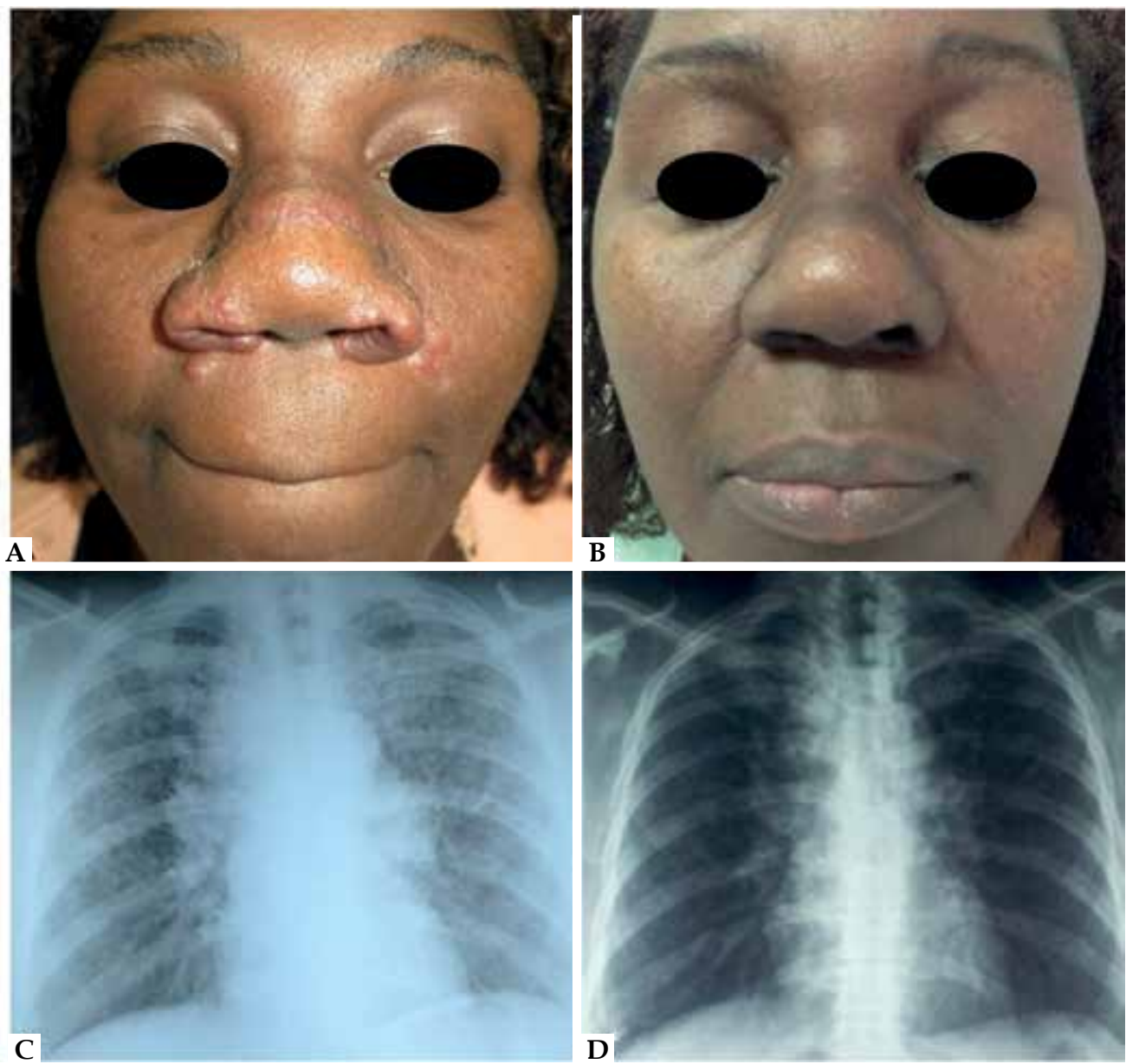

Figure 3: : A and B) Nasal region before and after 30 days of treatment. $C$ and D) X-ray of thorax before and after 30 days of treatment 
works as an immunological stimulant factor in cases of sarcoidosis. INF, in this context, would act as an amplifier or catalyzer of the inappropriate immunological response. ${ }^{5,6,7}$ Currently it is discussed whether there is statistical significance in this relation or if it is only pure coincidence. Isolating HCV in the lesional tissue is one more step in this investigation. However, through PCR, it is not possible to attest in which tissue compartment the microorganism can be found. It is known that the HCV circulates in a systemic fashion, possibly being present in local vasculature. It is worth remembering that analogous studies about lichen planus demonstrated the presence of $\mathrm{HCV}$ through immunohistochemistry in keratinocytes in lichenoid tissue. suggesting its direct participation in the pathogenic process. ${ }^{8}$ Therefore, new research including electronic microscopy or immunohistochemistry techniques may bring answers to some of these questions.

Nevertheless, diagnosis of sarcoidosis is still by exclusion, with histopathology being fundamental in the majority of cases, especially after ruling out infectious granulomas. Inclusion bodies are frequently found in sardoisis granulomas and in other sarcoid granulomatoses, such as foreign body granulomas, and are not specific. Of these, we highlight asteroid corpuscles, which present as eosinophilic, star-shaped inclusions, with diameter between $10 \mu \mathrm{m}$ and $15 \mu \mathrm{m}$, found inside multinucleated giant cells. ${ }^{3}$
Regarding cutaneous lesions in this case, the subcutaneous ones stand out. Described by Darier and Roussy in 1906, they are considered rare manifestations, occurring in only $2 \%$ of individuals with sarcoidosis. ${ }^{9}$ Other typical manifestations of the associated disease were central for its recognition. When isolated, sarcoid subcutaneous nodules become a true diagnostic challenge.

In the treatment of sarcoidosis, few drugs have a well-established benefit as antimalarials, corticotherapy and methotrexate. Others are cited as beneficial in small series of cases. ${ }^{1,3}$ Nevertheless, in patients with $\mathrm{CHC}$ we found restrictions to some drugs, due to their hepatotoxicity, like methotrexate. Prednisone had already been used previously, in recommended dosage, without adequate results. Consequently the option was made for its association with colchicine, due to its anti-inflammatory action and the possibility of using it in compensated hepatopathy carriers, obtaining a good result. Several reports indicate the efficacy of colchicine in sarcoid arthritis and Wise described good response in a case of lúpus pernio. ${ }^{10}$

With this case report, we aimed to illustrate the occurrence of sarcoidosis in a patient with $\mathrm{CHC}$, bringing up the discussion about the association of these diseases based on several studies published in the literature.]

\section{REFERENCES}

1. Iannuzzi MC, Rybicki BA, Teirstein AS. Sarcoidosis. N Engl J Med. 2007;357:215365.

2. Daldon PEC, Arruda LHF. Noninfectious granulomas: sarcoidosis. An Bras Dermatol. 2007;82:559-71.

3. Haimovic A, Sanchez M, Judson MA, Prystowsky S. Sarcoidosis: a comprehensive review and update for the dermatologist: part I. Cutaneous disease. J Am Acad Dermatol. 2012;66:699.e1-18.

4. Maticic M, Poljak M, Lunder T, Rener-Sitar K, Stojanovic L. Lichen planus and other cutaneous manifestations in chronic hepatitis $\mathrm{C}$ : pre- and post-interferonbased treatment prevalence vary in a cohort of patients from low hepatitis $\mathrm{C}$ virus endemic area. J Eur Acad Dermatol Venereol. 2008;22:779-88.

5. Ramos-Casals M, Mañá J, Nardi N, Brito-Zerón P, Xaubet A, Sánchez-Tapias JM, et al. Sarcoidosis in patients with chronic hepatitis $C$ virus infection: analysis of 68 cases. Medicine (Baltimore). 2005;84:69-80.

6. Gayet AR, Plaisance P, Bergmann JF, Mouly S. Development of sarcoidosis following completion of treatment for hepatitis $C$ with pegylated interferon\{alpha\}2a and ribavirin: a case report and literature review. Clin Med Res. 2010;8:163-7.

7. Faurie P, Broussolle C, Zoulim F, Trepo C, Sève P. Sarcoidosis and hepatitis C: clinical description of 11 cases. Eur J Gastroenterol Hepatol. 2010;22:967-72.
8. Lazaro P, Olalquiaga J, Bartolomé J, Ortiz-Movilla N, Rodríguez-Iñigo E, Pardo M, et al. Detection of hepatitis $C$ virus RNA and core protein in keratinocytes from patients with cutaneous lichen planusand chronic hepatitis C. J Invest Dermatol. 2002;119:798-803.

9. Dalle Vedove C, Colato C, Girolomoni G. Subcutaneous sarcoidosis: report of two cases and review of the literature. Clin Rheumatol. 2011;30:1123-8.

10. Wise RD. Clinical resolution of facial cutaneous sarcoidosis with systemic colchicine and a topical corticosteroid ointment. Compr Ther. 2008;34:105-10.

\author{
MAILING ADDRESS: \\ Eduardo Guimarães Pereira \\ Rua Mariz e Barros, 775 \\ Tijuca \\ 20.270-004 - Rio de Janeiro - RJ \\ Brazil \\ E-mail: guimaraesegp@gmail.com
}

How to cite this article: Pereira EG, Guimarães TF, Bottino CB, D'Acri AM, Lima RB, Martins CJ. Sarcoidosis and chronic hepatitis C: treatment with prednisone and colchicine. 2016;91(2):231-4. 